\section{Public engagement means listening as well as talking}

\begin{abstract}
SIR — The Editorial 'Enough talk already'
(Nature 448, 1-2; 2007) concludes that governments should respond to the public concern expressed in engagement exercises, and invest in research on the health and environmental risks of nanotechnologies. I agree. I would, however, suggest that this is not enough.
\end{abstract}

Although we need more scientific research on the risks of nanotechnologies, we also need to encourage broader dialogue on notions of progress, quality of life, human needs and our visions of the future - both with and without nanotechnology.

In the social sciences, the concept of uncertainty has been extended beyond that of risk and a lack of research. First, within complex, open and interacting social and natural systems, there is an inherent and irreducible form of uncertainty that prevents the full range of impacts being delimited. An additional form of uncertainty results from the diverse values, interests and positions held on questions such as what actually constitutes social and environmental health. Finally, given the novel properties used within nanotechnologies, there will also inevitably be novel impacts that we are currently simply ignorant about. This is ignorance about the right questions to ask, rather than ignorance about the answers.

This means that we need a broader dialogue to take place, about the real-life value of potential applications coming from nanotechnology. Otherwise we risk falling into the trap of believing we can base decisions about nanotechnologies on an assessment of their potential impacts alone, disregarding our values in the face of multiple forms of uncertainty.

Public-engagement exercises can begin this kind of dialogue - but not if their purpose is simply building public trust in order to win acceptance of potentially controversial technologies.

\section{Fern Wickson}

Centre for the Study of the Sciences and the Humanities, University of Bergen, PO Box 7800, 5020 Bergen, Norway

\section{Public engagement: both sides need to be realistic}

SIR - Your Editorial on engaging the public over nanotechnology ('Enough talk already' Nature 448, 1-2; 2007) captured many of the key achievements stemming from the convergence of high technology and new democratic practice, as well as the challenges still faced.

As director of the public-participation organization Involve (www.involve.org.uk), I would like to add that we need a more mature relationship between science and society, whereby both sides are open about their concerns and aspirations and realistic about what they can offer.

Too often science is equated to evidence. But having, in an earlier role, commissioned scientific research for the UK Department for Environment, Food and Rural Affairs, I am aware that the answer you get can depend to a large extent on who does the research and how their contract is configured. Because trust is built through experiencing openness and honesty, the public need to understand science, warts and all.

A good start would be to move away from portraying narrowly calculated technological risks as accepted facts (when they rarely are) to being open about the great expanse of uncertainty that scientists are constantly navigating.

Science is almost always a journey into the unknown. That includes unpredictable benefits and uncertain costs.

Richard Wilson

Involve, 212 High Holborn, London WC1V 7BF, UK

\section{Physics Nobels should favour inventions}

SIR - Alfred Nobel left a fortune to finance annual prizes to be awarded "to those who, during the preceding year, shall have conferred the greatest benefit on mankind". One part, he stated, should be given "to the person who shall have made the most important discovery or invention within the field of physics".

Nobel did not emphasize discoveries. But these are much more respected by the scientific community than are inventions: $77 \%$ of Nobel prizes in physics have been given to discoveries, compared with only $23 \%$ to inventions.

This emphasis on discoveries has moved the Nobel prize away from its original intention of rewarding the greatest contribution to society in the preceding year.

Discoveries and inventions depend on each other. Many discoveries were only made possible through the invention of certain measurement instruments, and without earlier theories, many inventions would have been inconceivable.

The fundamental difference between the two, however, is that the result of an invention is typically an artefact or process, whereas a discovery is an abstract theory. Although both require prior theories and a process of experimentation, and both have a utilitarian function, discoveries aim to be as general as possible, whereas inventions strive to be concrete.

A closer look at the 17 inventions that won
Nobel prizes before 2005 reveals that 11 of them (64\%) are measurement instruments, for example the scanning tunnelling microscope.

Only three winning inventions have had direct practical applications to society: the gas regulator-controlled buoys made by Nils Gustaf Dalén were subsequently used in lighthouses; the transistor invented by John Bardeen, Walter Brattain and William Shockley is widely used in electronic devices; and Jack Kilby's work on the integrated circuit led to the development of personal computers.

Awarding more Nobel prizes for inventions would encourage inventors to tackle important problems such as global warming or the gap between the developed and developing worlds. The award given to Kilby for the development of the integrated circuit is a good example.

The invention of the electric telephone, first patented by Graham Bell, was a missed opportunity for a Nobel prize to acknowledge an invention that has brought the world closer together. But there is still hope for Tim Berners-Lee's invention of the World Wide Web.

Christoph Bartneck, Matthias Rauterberg Department of Industrial Design, Eindhoven University of Technology, Den Dolech 2, $5600 \mathrm{MB}$ Eindhoven, The Netherlands

\section{Traditional remedies put animal species at risk}

SIR — Both your Editorial 'Hard to swallow' and your News Feature 'A culture in the balance' on traditional Chinese medicine (Nature 448, 106 and 126-128; 2007) focused on herbal remedies. However, although herbal formulations make up the bulk of traditional Chinese medicine, many animalbased remedies are also used.

These often expensive remedies are increasingly sought after in China, and although only a small proportion of the tissue used comes from endangered species such as rhinoceroses and tigers, the demand is having destructive effects on many organisms. In particular, populations of many turtle and tortoise species, both in China and around the world, are declining rapidly as they are captured in the wild and their shells ground into a jelly that is thought to cure diseases such as cancer.

The ecological effects of the use of wildcaught animal tissues as part of traditional Chinese medicine are an important issue that must be part of any discussion of its merits and disadvantages.

Margaret B. Murphy

Department of Biology and Chemistry,

City University of Hong Kong,

83 Tat Chee Avenue, Kowloon, Hong Kong 